\title{
Aproximaciones a una interpretación de la historia de la educación en Colombia ${ }^{1}$
}

\author{
Camilo Andrés Medina² \\ Corporación Universitaria Minuto de Dios, Bogotá, Colombia ${ }^{3}$ \\ cmedina800@gmail.com
}

1 Artículo de investigación derivado de la investigación para la tesis de Maestría en Historia de América Latina, Universidad Pablo de Olavide, España.

2 Magister en Historia de América Latina, Universidad Pablo de Olavide.

3 Docente-Investigador 


\section{Aproximaciones a una interpretación de la historia de la educación en Colombia}

\section{Resumen}

El presente artículo parte de los estudios realizados sobre educación y pedagogía en Colombia. Sin embargo, nuestra historia es en gran parte la transmisión de la cultura occidental, cultura que solo pudo ser transmitida por medio del aprendizaje y transformada, de igual forma, por las influencias que en los territorios americanos existían. Transformando, mutando y desapareciendo muchas de las tradiciones, lo que nos hace pensar que una sociedad no puede sobrevivir sin un arraigo o como lo mencionamos anteriormente, debe mutar para que sus integrantes no se dispersen ni se olviden, transmitiendo así a las generaciones jóvenes toda la riqueza que guardan, perpetuando las habilidades que hacen posible la vida del colectivo.

Palabras clave: Educación en Colombia, Historia y educación, perspectiva histórica

\section{Approaches to an interpretation of the history of education in Colombia}

\section{Abstract}

This article is result of studies on education and pedagogy in Colombia. However, our history is more of a transmission of Western culture which could only be transmitted by means of learning and transformed in the same way by influences that existed in the American territories. Transforming, mutating and disappearing many of their traditions, what makes us think that a society cannot survive without some roots or as we mentioned previously must mutate so that its members do not disperse nor forget, thus transmitting to young generations the wealth enshrined in culture and so perpetuating the skills that permits collective life to thrive.

Keywords: Education in Colombia, history and Education, historical perspective

\section{Aproximações a uma interpretação da história da educação na Colômbia}

\section{Resumo}

O presente artigo está baseado nos estudos realizados sobre educação e pedagogia na Colômbia. Entretanto, nossa história é em grande parte a transmissão da cultura de Ocidente, cultura que só pôde ser transmitida mediante o ensino e que foi transformada pelas influências que existiam nos territórios americanos. $\mathrm{O}$ que transformou, mudou e fiz desaparecer muitas das tradições; isso faz que pensemos que a sociedade não pode sobreviver sem uma raiz ou, como já mencionamos, ela deve mudar para que seus integrantes não se dispersem nem se esqueçam, transmitindo assim às gerações futuras toda a riqueza que possuem, conservando as habilidades que permitem a vida do coletivo.

Palavras chave: Educação na Colômbia, história y educação, abordagem histórica 
Es comprobable que las culturas precolombinas no tenían escuelas formales. Eran educados al lado de los padres u otros adultos elegidos por la comunidad para este oficio, progresivamente los niños se presentaban a la vida entre los adultos. Se podría afirmar con el ejemplo, que la educación se presenta en diferentes formas y categorías, siendo en esencia, la transmisión de la cultura de un grupo generación tras generación, desarrollando habilidades y técnicas que condicionan la supervivencia del grupo. La educación cultural como podríamos llamar a la formación en las sociedades indígenas es diferente a la educación institucional.

Son varios los tópicos que rodean la formación de los ciudadanos de la República en los diferentes periodos de nuestra historia. El debate de los distintos actores terminaba en el ágora, sin un posible remedio, conciliar los temas académicos y la fe, resultó aún más complejo cuando se vinculaba como casi siempre los temas del mercado y la profunda crisis económica que vivía el Estado. Ni los intelectuales de la época, como tampoco los presidentes y mucho menos el pueblo y su élite, alcanzaron a pretender una educación acorde a las necesidades del país, la educación al servicio de la obediencia y en especial de la Ley, condenaban a un más a la nación, ya sometida por las amplias diferencias de clase, a una ignorante democracia, donde en escena la tabla moral y el estado se batirían en duelo contra las riñas de gallos y las corridas de toros ${ }^{4}$. Para la política nacional y sus representantes de cara al desarrollo social, es también importante el desarrollo particular, el cual debe ser impartido en diferentes grados según la cultura social, en mayor o menor medida (Jaramillo, J, 2002, p.272).

El siglo XIX se caracterizó por el constante conflicto entre los partidos políticos y la relación con la iglesia católica, identificando el modelo educativo con el vencedor de una guerra o con el partido de turno, así las cosas cada gobernante traía consigo un paquete de reformas a la instrucción de los ciudadanos. Las reformas más influyentes tuvieron su inicio en el año de 1850 con la reforma de los liberales. Iniciando en la descentralización administrativa, fiscal y generalizando la instrucción. En 1870 se ordenó por medio del decreto federal que la educación fuera gratuita, obligatoria y laica supervisada por el gobierno central, quien tendría sobre él la autorización de los planes de estudio entre otros efectos administrativos.

Pero dentro de los planes de gobernantes y gobernados, se encontraban las posibles acciones que llevarían al país a una reforma sustancial en la educación y las relaciones entre los ciudadanos. Para ello se realizaron cambios desde los cuerpos administrativos hasta lo pedagógico, de lo administrativo a lo social, concentrando

4 La cooperación del colectivo proviene de un estímulo e iniciativa individual, en donde las garantías son producto del trabajo de la sociedad y no son solo responsabilidad del estado. La política regeneradora de Núñez cree firmemente en el interés privado, aun sin contar con una experiencia industrial que confirme que culturalmente la ciudadanía es capaz de asumir el riesgo de un proceso de industrialización (Jaramillo, J, 2002). 
sus acciones en el poder central con efectos matizadamente obligatorios sobre toda la población en general. Ese cambio es posible en la evolución política de su gestor, Rafael Núñez ${ }^{5}$ había logrado comprender que para realizar cualquier cambio social debía contar con la unidad vital del pueblo. Siendo la institución católica el elemento de unidad, no podía desprender sus planes del consentimiento, aceptación y participación de la mencionada institución.

Casualmente las ideas regeneradoras de Núñez, eran muy similares a las del conservador Miguel Antonio Caro, para quien la República le reconoce a la nación, una sola religión para todos los colombianos, punto de partida para conseguir la paz y la concordia. En segundo lugar, el papel relevante que ocupa la institución eclesiástica había que reconocerlo políticamente, para ello se le otorga la personería jurídica, con la cual se establecería un constante dialogo entre el ejecutivo y los representantes de la santa sede, al igual que se pondrían los términos en las relaciones entre el estado y la Iglesia dado su carácter independiente. En el título IV de la Constitución de 1886 se reafirma y delimita la relación entre la iglesia y el estado, enfatizando la incompatibilidad en el desempeño de cargos públicos, lo que pone fin a las ambiciones personales del sacerdocio y a las intenciones de expansión del poder romano.

Como el primer paso para llevar a la nación un nivel óptimo de educación, organiza la entidad que denomina Instrucción Pública Nacional, facultada y reglamentada en la Ley del 23 de Agosto de 18806; la cual rompe con el sistema tradicional de ver la organización de las instituciones del Estado, pasando por un control total sin autonomía significativa, pero con el poder de cambiar y reglamentar a gusto del gobierno central, según la intervención que el ejecutivo considerará conveniente.

Al concentrar la educación en el nuevo ente encargado de la instrucción de

5 Rafael Núñez. Presidente en cuatro oportunidades logró consolidar un proyecto político en materia de educación.

6 Ley del 23 de Agosto de 1880, Dentro de esta Ley los artículos más significativos. Art.- $1^{\circ}$ Corresponde al Poder Ejecutivo la organización i dirección de la enseñanza pública - oficial en todos sus ramos. Art.- $2^{\circ}$ La instrucción pública que costea la Nación se divide en primaria, secundaria i profesional. Art.- $3^{\circ}$ La instrucción primaria comprende la que, de conformidad con las leyes y los decretos vijentes, se da en la República en las Escuelas normales i primarias de todos los grados. Art.- $4^{\circ}$ La instrucción secundaria o media, comprende los ramos de literatura i ciencias aplicadas que constituyen el cuadro de estudios de la Escuela de Literatura i Filosofía de la Universidad Nacional. Esta enseñanza tiene por objeto preparar a los educandos con estudios completos, para las carreras profesionales. Art.- $5^{\circ}$ La instrucción professional comprende las enseñanzas de Ciencias naturales, de Ingeniería Civil i Militar, de agricultura, de Artes i oficios, de Ciencias Políticas, de Derecho, de Medicina i de Náutica. Art.- $22^{\circ}$ Quedan derogadas la lei 26 de 1876, "que organiza la Universidad Nacional," la de 30 de mayo de 1868," sobre instrucción pública, "la de 2 de Julio de 1870," que autoriza al Poder Ejecutivo para organizar la Instrucción Pública primaria," la 69 de 1877" que crea la Escuela de Injeniería civil i Militar" i la lei 4º de 1874; i reformadas la 78 de1873 i el artículo 1179 del Código Fiscal. 
los educandos, se logra al igual concentrar en la capital la enseñanza secundaria y profesional, exclusive la formación náutica que por sede se ubicaba en Cartagena. Bogotá pasa a ser la ciudad educadora de las masas que querían alcanzar una educación mayor, la reforma también exige de esta una mayor infraestructura en cuanto a su mobiliario para recibir a los nuevos estudiantes. Y concentrarlos alrededor de los centros de formación. Así también, la Universidad Nacional sufre un primer cambio, concentrando en ella todas las facultades, que en años anteriores se encontraran desprendidas o autónomas, al agruparlas se asegura poder controlar la formación impartida en el claustro; acorde a la concepción de estado y política que impondría después de la constituyente.

En la construcción de la unidad nacional por parte del estado, supone de él la inversión en la unificación del territorio, la disposición de recursos para cubrir los gastos que sugiere la integración de toda la ciudadanía, la construcción de infraestructuras que conecten las regiones y la imposición de la autoridad central que dirige, organiza, emplea, delega y ordena sobre los intereses de la nación y del fortalecimiento del Estado.

Los cambios ya preparaban a la República para la irrupción de otra transformación en la enseñanza; constituían la plataforma para la introducción de la educación religiosa en todos los niveles, siendo también el punto de partida de las reformas educativas de 1886 y 1887 . La entrega de la educación y de los claustros de formación a la iglesia pronto trajo consecuencias en la sociedad, declarando abiertamente su rechazo por todo lo que se asumiera como ideología liberal y desterrando toda influencia francesa de la formación de los futuros ciudadanos.

El alto estatus obtenido en el Concordato ubica a la institución en un lugar de privilegio, incluso ante el mismo Estado que se había encargado de darle un alto reconocimiento, dejando ver las múltiples limitaciones que poseía el gobierno en términos de cobertura y de autoridad. Demostrando al ejecutivo colombiano el amplio poder de influencia que ejercía sobre los nacionales, legitimidad que aún buscaba el mandato, permitiéndose usar los amplios recursos de los cuales gozaba y podía usar a su discreción, siendo también herramienta el disponer de un número suficiente de miembros que garantizaba una cobertura total sobre la extensión del territorio. Frente al limitado accionar de las fuerzas del poder central, carente de recursos y de personal.

Pese al control del estado, Colombia en el siglo XX era una de las naciones de América más rezagadas en materia de cobertura, calidad e investigación, producto de la extrema pobreza, un incipiente desarrollo económico en materia de exportaciones, el sostenido conflicto entre los intereses de gobierno y religión. Las reformas ejecutadas por los gobiernos liberales entre los años 1930 a 1945 denotaron una baja en el analfabetismo, seguía siendo incipiente pues este reflejaba 
solo un $10 \%$ del total 7 . De aquí en adelante la migración de campesinos a los cascos urbanos incide significativamente, teniendo en cuenta que los gobiernos hasta la década del 50 no crecían acorde con las necesidades de los nuevos pobladores.

Sin embargo, las medidas iniciadas por el Frente Nacional generaron un crecimiento significativo en el número de matriculados que para el año de 1957 era de 1.500.000 estudiantes a 4.000.000 en el año 1970. Aunado por el incremento de instituciones privadas que ofrecían calidad, en un mercado que poco a poco iba perdiendo el estado al no poseer una ruta clara que identificara un sistema educativo coordinado con los diferentes niveles de la educación formal.

A falta de recursos y medidas claras en la política nacional de educación en la década del 80 el impulso educativo tuvo un freno considerable, comparado con el crecimiento de la población en las ciudades. Reforzado por los recortes presupuestales otorgados al ministerio de educación, que en el año de 1970 fueran del $22 \%$ del gasto nacional en el año 2.000 se había recortado apenas al $12 \%$, trasladando esos recursos al gasto en seguridad, salud y pensiones, que ocuparon un puesto prioritario en la agenda económica del país.

Con la entrada en vigencia de la nueva constitución (Carta política de 1991), se pretendió garantizar el acceso masivo de niños entre los 7 y 15 años a la educación básica y secundaria ${ }^{8}$, garantizando mayor cobertura, recursos y calidad sobre las competencias que pretende desarrollar. Desafortunadamente, a pesar que la educación es un derecho de todos los colombianos, los actos legislativos encargados de regular la misión de esta, en contraposición a su espíritu e intereses de la nación; la educación ha sufrido de múltiples recortes en diferentes niveles. La desprofesionalización de la educación, la descentralización del sistema educativo descargado la responsabilidad fiscal en los gobiernos departamentales ${ }^{9}$, concentrando los esfuerzos en la inversión en infraestructura escolar obviando el capital humano, es hoy el talón de Aquiles de un sistema que naufraga entre la cantidad y la calidad.

\section{Acceso y masificación de la educación}

Durante el siglo XIX las estadísticas no reflejan una realidad aproximada en su primera mitad dado los constantes conflictos y cambios en la política nacional, sin embargo, es hasta finales del siglo cuando la restructuración del estado, nos permite ver en mayor detalle los efectos en la educación programas y acceso.

7 Salomón Kalmanovitz (2007) Afirma que el analfabetismo cae del 50 al 40\% donde se calcula que en promedio cada ciudadano de la república solo alcazaba el grado 4 de formación básica

8 Constitución Política de Colombia de 1991.

9 Ley 60 de 1993 que definió las potestades y competencias en las áreas de Educación, Salud y distribución de recursos. 
La constitución del 86 pone de manifiesto la relación entre el estado y la institución católica, dándole a esta última la potestad de dirigir la formación de los ciudadanos en materia moral, de control social y en la instrucción escolar. La Constitución declara en el artículo 41 sobre la instrucción primaria, su gratuidad y no obligatoriedad, acorde al ideal de Miguel Antonio Caro, para quien la Regeneración propuso que la relaciones entre el Estado y los individuos, provienen de la iniciativa individual.

En consecuencia a las normas dictadas por el ejecutivo se propusieron tres tipos de escuelas divididas por género, las escuelas para señoritas, para hombres y las alternadas, con lo cual se pretendía cubrir las necesidades formativas de los jóvenes bogotanos, implicando una estricta vigilancia y un minucioso control por parte del órgano de Instrucción Pública.

La formación femenina que desde comienzos del siglo había estado dedicada a la enseñanza de la economía doméstica y las manualidades. En la década de 1870 amplía sus áreas de enseñanza de acuerdo a la entidad instruccionista y a las disposiciones del poder central; así en la formación básica se incluyeron asignaturas propias de la lengua como: lectura, escritura, aritmética, ejercicios de composición, normas generales de higiene, historia y geografía (Zuluaga, 2002). En la formación media, adicionalmente a las asignaturas mencionadas, estudiaban elementos de álgebra, geometría, nociones de física, mecánica, química, historia natural, fisiología e higiene, elementos de cosmografía ${ }^{10}$, geografía general y específica de Colombia al igual que su historia. Estas asignaturas se repartían en menor intensidad respecto a la enseñanza del trabajo con agujas, economía doméstica y otras prácticas consideradas importantes para la actividad femenina. La enseñanza religiosa impuesta por el gobierno regenerador, trae nuevas asignaturas obligatorias entre las cuales se cuenta la historia sagrada y la urbanidad. Ampliando aún más la carga para la educación femenina, se introducen materias complementarias a las manualidades y las labores con agujas, costura, bordados, tejidos y corte de trajes, corresponde a la actividad formativa.

José Liborio de la Serda incluye en el plan de estudios cambios acordes a los niveles de enseñanza, en los cuales la instrucción se regula por las edades, manteniendo un promedio de aprendizajes, en virtud del desarrollo integral de los estudiantes en la moral, el cuidado del hogar y costumbres saludables. Cumpliendo con un horario se exige tanto a las niñas como a los hombres asistir a la escuela de lunes a sábado de 7 de la mañana a 2 de la tarde ${ }^{11}$.

10 Cosmografía se define como el estudio que antecede la ciencia de las astronomía, esta definición aparece por primera vez en la serie de libros escritos por Claudio Tolomeo (100 a 170 a.n.e), el octavo libro conocido como GEOGRAPHIA es el que describe las cartas geográficas y el lugar de la tierra en el universo explicando a través de mapas, la matemática geográfica entre otros temas relacionados con los cálculos latitudinales del globo terráqueo. Es Jacobo Angelus de Scarperia quien al realizar la traducción del texto (1406), cambia el nombre a Cosmographia.

11 Decreto 99 de 1886, 17 de febrero, por el cual se organiza la Instrucción pública, contenidos formativos, 
El sostenimiento de las escuelas para niñas, se cargó a los distritos, con la obligación de abrir una escuela elemental en cada uno, en caso de que los recursos no fueran suficientes la municipalidad asumía el costo y mantenimiento del centro de formación. En caso que los distritos no lograran poder sostener las escuelas, ellas serían trasladadas a lugares cercanos, que pudieran costear dichos gastos. En determinados casos las escuelas públicas femeninas, podían ser suprimidas, siempre y cuando la educación privada pudiera satisfacer las necesidades de educación ${ }^{12}$. Para el año de 1893 el decreto 429, establece que los ciudadanos de cada distrito con sus aportes, sostuvieran el número de escuelas primarias suficientes para la formación de las niñas entre los 7 a 15 años de edad. Para que la mayoría pudiera acceder a la formación gratuita ${ }^{13}$.

\section{Educación primaria y secundaria.}

La primera formación que recibía una persona constituía el paso inicial en el proyecto regeneracionista y el que más discusiones llevaría, para los administradores públicos. Don Higinio Cualla García, primo de Núñez asume la alcaldía de la capital en 1884, durando en el cargo hasta 1889. Con importantes logros para la ciudad; como el alcantarillado, las líneas del tranvía y la higiene pública. Este cartagenero fiel a sus convicciones y al ideal regenerador, prendió los motores de la educación llevando a la ciudad a un liderazgo escolar importante, al igual que elevó el número de estudiantes en las aulas de formación.

Cuadro 1. Cundinamarca, escuelas frente al país

\begin{tabular}{|c|c|c|c|c|}
\hline Estado & \multicolumn{2}{|c|}{ Escuelas Primarias } & \multicolumn{2}{|c|}{ Estudiantes } \\
\hline Año & 1880 & 1883 & 1880 & 1883 \\
\hline Cundinamarca & 228 & 164 & 13.829 & 10.624 \\
\hline $\begin{array}{c}\text { Total en } \\
\text { la unión }\end{array}$ & 1.395 & 1.297 & 71.501 & 68.268 \\
\hline
\end{tabular}

Fuente: Fundación Misión Colombia,1988

El cuadro nos da una idea sobre la situación de la educación en el departamento, frente al resto del país. De las cuales y en la medida que la inestabilidad política se

acordes a las indicaciones de la iglesia y los valores que se consideraban debía tener cada estudiante, formada en una escuela y dotada de todas las bondades que Dios a través de la Iglesia le había encomendado.

12 Decreto 595 de 1886. Código de Instrucción pública. p. 1146-1151.

13 Decreto 429 de enero de 1893. 
agudizaba, la escuela perdía territorio y cobertura, con directos efectos sobre la ciudad y sus inmediatas áreas rurales, el reclutamiento de menores fue uno de los factores que influyeran directamente en el número de asistentes. Por otro lado, con la escasez de alumnos las edificaciones escolares se redujeron a lugares destinados para la guerra.

Así con la nueva administración el panorama inicia un proceso favorable para la educación pública de los más pobres, la infraestructura de las escuelas es solo uno de los contrastes, con los propósitos de llevar una formación adecuada, en improvisados salones de alquiler, cargados de forzadas transformaciones, los lugares no llegaban a cumplir con ninguna de las especificaciones, para una adecuada arquitectura escolar (Zuluaga, 2002). La información recolectada también nos aporta información valiosa para la interpretación y entendimiento de los cambios y sus efectos sobre las gentes. Sin embargo todos los datos recolectados sobre educación e instrucción pública, en los diferentes niveles deformación, pertenecen a información obtenida deaños posteriores.

"La Instrucción Pública primaria para niños en los planteles, con asistencia: escuelas primarias, 530; escuela de San Vicente, 310; Asilo de niños, 150; escuela anexa a la Normal de Institutores, 42. En total 1032. Hay que agregar la enseñanza primaria en los cuarteles para 1500 reclutas." $^{\prime}$

"Instrucción Pública primaria para niñas en los siguientes planteles: escuelas primarias, 506; Escuela anexa a la Normal de Institutoras, 6. En total 567 niñas."

“Enseñanza primaria para niños en los siguientes planteles privados: escuelas Hermanas de la Caridad, 145; escuela Niño Dios, 30; Liceo infantil, 44; 9 escuelas, 203. En total 422 niños" (Martinez, 1976).

“Enseñanza primaria para niñas en los siguientes planteles privados:Escuela Hermanas de la Caridad, 393; Casa de Jesús, Maria y José, 80; escuela de Sanvicente, 78; 22 escuelas varias, 445. En total 996 niñas" (Martinez, 1976).

Los niveles de formación entre los colombianos eran muy deficientes. Este margen hace pensar que el acceso usado se encontraba en minorías privilegiadas, haciendo mayor el analfabetismo, afirmando el avanzado estado de pobreza, y evidenciando con más fuerza la condición de ruralidad de los ciudadanos de la capital, que probablemente encontraban como escuela para sus hijos el patio de la casa, un huerto o un taller, recibiendo allí la formación necesaria para una vida medianamente productiva. Los pocos grupos que accedían a la formación no contaban con gran material de apoyo, haciendo aún más compleja su formación, acudían a las herramientas que la mendicidad y gestión propia les permitía.

Coincidencialmente al ya oscuro panorama de la formación en el país hay que añadirle otro elemento aún más feroz. Los intereses de la oposición también debilitaban la ya roída estructura educativa del país, inaugurando escuelas privadas, cumpliendo con las obligaciones nacionales, pero con una fuerte influencia radical, 
las asignaturas llevaban entre sus líneas la suficiente información ideológica, estrategia destinada a formar soldados de oposición, que pudieran llenar las filas del Ilamado guerrerismo partidista. Este ideario llevo a que la educación se fuera consolidando en el sector de lo privado, haciendo languidecer la formación pública gratuita. Entre 1888 y 1894 la fundación de colegios privados ya arroja un indicador que muestra su ligera pero constante aceptación (Fundación, 1988).

La precaria transformación de la ciudad en Colombia, al igual que el hacinamiento de sus habitantes, las escuelas públicas retrataban ese incomodo panorama habitacional. En los centros particulares se educaban un promedio de 28 niños, 38 niñas por escuela, en el escenario oficial el número alcanzaba 63 estudiantes por cada director de institución para varones. Las escuelas femeninas, con carácter privado, albergaban en sus aulas entre 20 y 25 estudiantes, acentuando el contraste a la formación pública o de caridad que se ofrecía por parte de la ciudad y de las religiosas, aglomerando hasta 150 alumnas en un cuarto. Demostrando las desfavorables condiciones para una buena formación y la arraigada diferencia entre las clases sociales. Brecha que daba a unos la educación casi personalizada con un alto nivel de dedicación y a otros los ponía en la calle de la mendicidad alfabética.

El compromiso del estado en la educación básica trató buenamente de cubrir los sectores más vulnerables, sin poderse desapegar de sus tareas, la formación fue llevada a más grupos sociales, entre ellos la educación primaria adulta. Definida como política oficial, pretendía mejorar el comportamiento de los pobres, pretendiendo a través de la formación, cambiar algunas de sus costumbres muy criticadas por las élites. En otro escenario de la ciudad, el proyecto educativo abordaba la formación cristiana de la mano de las prácticas religiosas que diariamente se impartían, con carácter obligatorio, se enseñaba, castellano, aritmética y a algunos tejidos en fique. Así, el panóptico constituía otro lugar de formación encargado por el estado a los sacerdotes, propendiendo por eliminar el crimen a través de la formación básica y el temor a Dios. Así como los condenados recibían formación, la soldadesca que conformaba los cuatro batallones de la ciudad eran instruidos en las materias fundamentales (Fundación, 1988).

La inversión en educación para las escuelas primarias y secundarias, frente al ingreso y gasto del PIB fue reducido significativamente a lo largo del siglo XX, difícilmente superó el 3\% del PIB, comparado, claro está, con la inversión y gasto administrativo del estado (Avella, 2009). Determinada por el incremento en las rentas del estado explicadas en los esporádicos auges económicos, estimulados por los cambios institucionales la apertura comercial y financiera. Influenciada por las fluctuaciones dadas por el auge del café y la exportación de oro (Ocampo, 2007).

La educación secundaria es el colofón de un plan abiertamente planeado para la oligarquía, hecho que dejo secuelas y enormes esfuerzos de padres e hijos que 
buscaron por todos los medios alcanzar las puertas de la formación secundaria. Paso para una vida más cómoda y en mejores condiciones dentro del creciente abismo social. Así como pasó en la educación primaria, la formación secundaría oficial perdió fuerza en la capital, que para el año de 1891 contaba en las instalaciones del Colegio Menor del Rosario con un número de estudiantes que rodeaba los 327, la Escuela normal de Institutores formaba 42 maestros, el Colegio militar Ilegaba a los 62, en la escuela de Bellas artes se preparaban 90 futuros artistas y en la Escuela de Música en la cual se formaban 60 hombres y 35 señoritas.

El género femenino recibía su formación en los colegios de La Merced, Nuestra Señora del Pilar y la Escuela Normal de Institutoras. Educando a un número en orden; 85, 40 y 64 alumnas. De las cuales las institutoras aplicarían posteriormente a los cargos de profesoras o institutrices particulares en casa de personalidades o familias distinguidas que así lo decidieren.

Dentro de su compromiso el Estado financiaba algunas instituciones dedicadas a enseñar a los hijos de artesanos y otros trabajadores, como lo eran la Escuela de Telegrafía, en la que sumados estudiaban 22 varones y 24 niñas en la sección femenina. El instituto Salesiano, educaba a 178 varones y se encontraba bajo la dirección de la Orden Salesiana. Al igual hizo su aporte en su momento el Instituto de Artesanos, con recursos públicos, la formación allí impartida iba dirigida a jóvenes y adultos. Recibiendo a sus estudiantes durante las primeras horas de la noche, se les instruía también en hilados, tejidos y tintorería, prácticas que realizaban durante el día en sus lugares de trabajo.

La educación secundaria privada, que por demás está decir, era más dedicada y fervorosa para sus estudiantes, en informe presentado, cuentan con 7 instituciones de formación secundaria, perteneciente al sector privado, de ellas la Universidad Republicana que impartía instrucción primaria, secundaria, a un número de 238 estudiantes, el informe cuenta también con un número impreciso de estudiantes en el Colegio Seminario, pues no se puede determinar cuántos eran seminaristas en estudios de secundaria, con los estudiantes matriculados de forma regular. Tampoco es posible determinar el número de estudiantes de secundaria y cuántos eran universitarios formados en la Universidad Católica. Así, el número de educandos puede llegar a 433 jóvenes en este nivel de formación repartidos en los 4 colegios de la ciudad. El grupo correspondiente al género femenino, comprende un número de 353 señoritas repartidas en 5 colegios, en número que va de integrar entre 7 estudiantes, hasta 28.

Marcando una notoria diferencia el Colegio Pestazlozziano que cuenta con 140 alumnas. Así podemos referenciar una diferencia en el acceso a la educación secundaria entre la formación oficial y la privada, donde en el primer sector asisten 1029 estudiantes, mientras que en el privado hay 1275 alumnos. 
La proporción de estudiantes matriculados a comienzo de siglo en la educación secundaria apenas superaba el $0.5 \%$ del total de la población, consecuentemente el aumento del número de estudiantes se mantuvo por debajo del 10\% hasta 1970 . Determinado por factores como la falta de profesores idóneos para la práctica educativa, los bajos sueldos, el número de maestros sin grado y la desproporción entre la presencia de escuelas normales en el área urbana frente a las enormes zonas rurales. Aunado por la falta de materiales que complementaran la actividad docente.

El cambio producido por la coalición bipartidista del Frente Nacional, que se interesa en la promoción y fortalecimiento de la industria pesada, que permitió introducir la Ley 111 de 1960 que nacionaliza el gasto para la educación fijándolo en el $10 \%$ del presupuesto nacional. Lo cual permitió un flujo constante entre los departamentos y el gobierno central para la financiación de la educación oficial en los municipios y las áreas rurales. El ingreso generado por esta política, junto a la migración del campo a la ciudad, propició el fortalecimiento y la construcción de instituciones donde albergar y formar a los nuevos residentes. Lo que permitió fortalecer la mano de obra en la industria que pedía mayor calidad y un número más alto de personal con estudios básico y técnico, que le diera competitividad y transformación en el creciente mercado nacional, que le abría paso a las mercancías producidas con los recursos y las materias primas propias.

Continuando con las reformas durante el gobierno de López Michelsen se pretende un nuevo intento por reorganizar el sistema educativo reformando y modernizando el MEN, logrando mayor énfasis en la educación pública. El número de docentes se incrementa con la puesta en marcha de programas de formación en ciencias de la educación, el auge en la demanda de las instituciones privadas y públicas. Para el año de 1975, la ley 43 promovió la mejora en los esquemas de la educación, transfiriendo la financiación al gobierno central; sin embargo, ello no logró controlar el desorden administrativo en la contratación de los maestros que suplirían las necesidades en las áreas apartadas y los cascos urbanos a lo largo y ancho de todo el territorio.

Los gobiernos de López Michelsen, Turbay y Betancur, demostraron una vez más, el atraso en términos educativos y política educativa, con el ánimo de dar un salto hacia la calidad las políticas se encaminaron al fortalecimiento de los INEM, que proporcionaría la educación necesaria para atender los requerimientos de la nación. Concomitante a estos cambios en cobertura se creó el Estatuto Docente en busca de mejores condiciones laborales, considerado este como el mayor logro de las recientes asociaciones de maestros. Lo que permitía no solo la consolidación de la profesión docente, la promoción, cualificación y el escalafonamiento, proporcionaba la participación en los cambios, propuestas y planificación de las políticas en la materia. 
Desde la adopción de Escuela Nueva en 1970, la educación vio por fin materializadas todas sus metas, hasta 1983 época en la que el estado colombiano inicia una fuerte campaña de control al gasto en la educación pública. Tendencia que se agudizaba con los efectos recesivos de una institucionalidad que observaba como sus ingresos desmejoraba junto a la cambiante economía mundial. Lo que incidió inmediatamente en los indicadores educativos.

Finalmente en el 91 como lo mencionamos en un comienzo la C.P, permite junto al Magisterio promulgar la Ley general de Educación de 1994, la cual establece la administración, dirección y financiación de la educación en Colombia, fundamentado en el artículo 72 de esta Ley se expide el decreto 1719 de 1995 por el cual se dictan las condiciones para los Planes Decenales de educación. Con los cuales se pretende la universalización de la educación en Colombia. 


\section{Referencias}

Aguilar, J., Betancourt, J. (2002). Construcción de una Cultura Democrática en Instituciones Educativas de Santa Fe de Bogotá. Santa Fe Bogotá: Idep Innove.

Álvarez, A. (2005).Poder. Democratización y Formación Ciudadana. Serie Convivencia y Democratización de la vida Escolar. Alcaldía de Santiago de Cali: Universidad del Valle..

Avella, M. (2009). El crecimiento del gasto público en colombia, 1925-2003, ¿una visión descriptiva à la wagner o à la peacock y wiseman?. Rev .econinst. [online]. Recuperado de http://www.scielo.org.co/scielo.php? script =sci_arttext\&pid=S 012459962009000100004\&lng=en\&nrm=iso $>$. ISSN 0124-5996.

Constitución política de Colombia. (1991) Santa Fe de Bogotá, Colombia.

Cubides, H. (2001). Gobierno Escolar: cultura y conflicto político en la escuela. Revista Nómadas.15:(23-36).

Frigerio, G., et al. (1997). Políticas Institucionales y Actores en Educación. Centro de Estudios Multidisciplinarios. Colección Reflexión y Debate. Buenos Aires: Ediciones Novedades Educativas.

Gómez, A. Hernando. Educación Agenda del Siglo XXI Hacia un Desarrollo Humano. Programa de Naciones Unidas para el desarrollo. Santa Fe de Bogotá:Tercer Mundo Editores. 1998. 366p

Fundación Misión Colombia. (1988). Historia de Bogotá, Tomo VI. Bogotá: Siglo XX.

Jaramillo, J. (1989). La educación durante los gobiernos liberales. 1930-1946. En: Tirado, A. (Ed.). Nueva Historia de Colombia. Tomo IV. Bogotá: Editorial Planeta.

Jaramillo, J. (2002). El pensamiento colombiano en el siglo XIX. Bogotá: ALFAOMEGA.

Kalmanovitz, S. (2007) La educación en el siglo XX. El Espectador, 14 de Abril de 2007.

Lebot, I. (1972). Elementos para la historia de la Educación en Colombia, en el siglo XX.En DANE. Boletín Mensual de Estadística. 249:. 135-141.

Lebot, I. (1985). Educación e ideología en Colombia. Medellín, Colombia: Editorial La Carreta.

Martínez, C. (1976), Sinopsis sobre su evolución urbana.Colección Historia. Bogotá: Escala Fondo editorial.

MINISTERIO DE EDUCACIÓN NACIONAL. Plan Decenal de Educación 1996 -2005. 390p

MINISTERIO DE EDUCACION NACIONAL. Programas estructurados nacionales para el desarrollo de las competencias ciudadanas. 2004. 290p

MINISTERIO DE EDUCACION NACIONAL. Reforma Educativa y Proyecto Educativo Nacional. Reinvención de la Institución Escolar. Informe al Congreso Nacional. 
Ocampo, J. (2007). Historia económica de Colombia. Bogotá: Planeta y Fedesarrollo.

Zuluaga, O. (2002). Historia de la Educación en Bogotá. Bogotá: Alcaldía mayor de Bogotá.

Recibido: 26 junio 2013

Aceptado: 28 agosto 2013

Cómo citar:

Medina, C.A. (2014). Aproximaciones a una interpretación de la

historia de la educación en Colombia. Praxis Pedagógica, 15, 141-155 